\title{
MULTIPACTING STUDY OF THE RF WINDOW AT THE ADVANCED PHOTON SOURCE*
}

\author{
J.J. Song", Argonne National Laboratory, IL
}

\begin{abstract}
Multipacting current can cause breakdowns in high power rf components such as input couplers, waveguide windows, and higher-order mode (HOM) dampers. To understand and prevent the loss of a ceramic window or an input coupler in the Advanced Photon Source (APS) storage ring rf cavity, the multipacting phenomenon is being investigated experimentally. This paper begins with a description of simple model, presents a hardware design, and concludes with measurement of multipacting. Multipacting is explored in conjunction with conditioning the cavities and interaction with the stored beam.
\end{abstract}

\section{INTRODUCTION}

The APS at Argonne National Laboratory is fully operational at $7-\mathrm{GeV}$ with a beam current up to $100 \mathrm{~mA}$ producing various types of $\mathrm{X}$-ray radiation. Since energy is lost due to synchrotron radiation, the storage ring (SR) rf system typically provides about $9.6 \mathrm{MeV} /$ turn at $100 \mathrm{~mA}$. The SR rf system consists of four sectors, each consisting of four single-cell cavities $(4 \times 4)$. The four rf sectors are powered by two 1-MW klystrons for 100-mA operation.

However, several ceramic windows have been damaged during full operation of the SR rf system. The main symptoms are higher temperature, beam loss due to pressure bursts, and arcing. The inspection revealed a copper coating inside the ceramic windows as well as vacuum leaks due to cracks and/or pinholes on the windows. To prevent further loss of a ceramic window/input coupler in the APS SR rf cavity, an experimental study of the multipacting phenomena on the cavity has been prepared.

\section{MULTIPACTING}

Multipacting is a well-known phenomenon frequently observed in rf systems such as rf cavities, HOM absorbers, and klystrons. When an rf field exists across a gap, an electron from one surface is accelerated toward the other surface. Upon impact, another electron may be released due to secondary electron emission. The secondary emission coefficient $\delta$ depends on the electron impact energy $E_{i}$ and properties of the surface material. If the secondary emission coefficient of the surface is high enough $(\delta>1)$ and the emitted electrons can be accelerated with a resonant field, this process can continue and lead to multipacting.

Work supported by U.S. Department of Energy, Office of Basic Energy Sciences under Contract No. W-31-109-ENG-38.

${ }^{\#}$ Email: jsong@aps.anl.gov
There has been a substantial investigation regarding this subject by others. Most of these concentrate on a simple 1-D model simulation or an experimental measurement with planar electrodes. R.A. Kirhek et al. [1] introduces a simple model as shown in Figure 1. In this model, the multipacting current due to the planer structure is added to the ordinary LRC circuit of the rf cavity in the typical representation. He addresses issues such as the multipacting saturation level, the multipacting mechanism, and the evolution of multipacting. On the other hand, D. Proch et al. [2] presents a systematic measurement of the rf multipacting current. The multipacting current between two planar electrodes of a specially designed $500-\mathrm{MHz}$ coaxial resonator was measured. Various measurements were made by changing the surface material and condition of the electrodes.

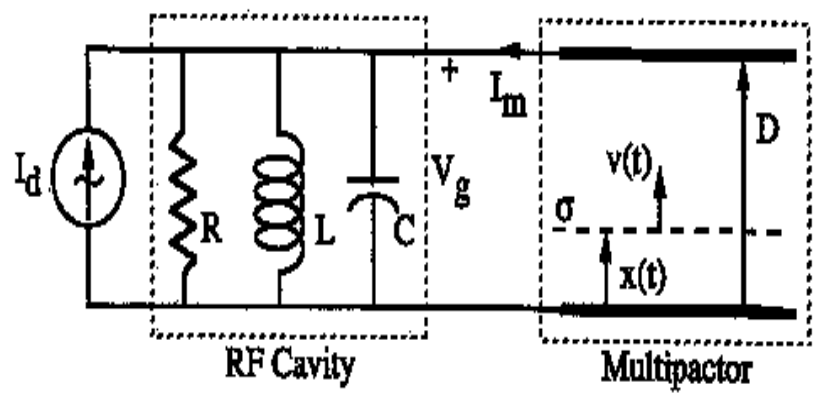

Figure 1: A simple model for interaction of multipacting with an rf cavity. The LRC circuit represents the rf cavity, loading by multipacting current $\mathrm{I}_{\mathrm{m}}$.

Multipacting is clearly undesirable. It results in the dissipation of large amounts of power and thereby generates destructive thermal-stress gradients. Furthermore, the power loss may occur in localized regions, leading to increased outgassing or evaporation of the material. Ultimately, the multipactor loading may become so great that the desired rf gap voltage cannot be reached or may cause significant reflection.

Unfortunately, few elements have low secondary emission characteristics. Moreover, many of these are unsuitable for vacuum and/or rf applications. Of particular interest to coupler windows for the APS SR rf cavity are the secondary emission coefficients of alumina and copper, which are $\delta_{\max }=2$ to 9 and 1.3 , respectively. To reduce the multipacting phenomenon, the rf windows at APS have been coated with titanium, using a simple deposition system before conditioning and operation [3]. 


\section{MEASUREMENT SETUP}

A prototype electron detector system (e-detector) was built, bench-tested, and connected to the APS SR rf cavity near the ceramic window to measure the secondary electron emission (SEE) current. This device is similar to the edetector initially developed at DESY [2].

The ceramic window allows for rf power to be transmitted from the WR2300 waveguide to the $352-\mathrm{MHz}$ singlecell cavity through an H-loop-type input coupler as shown in Figure 2. The field patterns are transformed from $\mathrm{TE}_{11}$ mode in the waveguide to $\mathrm{TM}_{010}$ mode in the cavity. While the electromagnetic field is transmitted through a coaxial input coupler, the field configuration appears to be a TEM type. The e-probe of the detector was embedded at the bottom of the input coupler near the window.

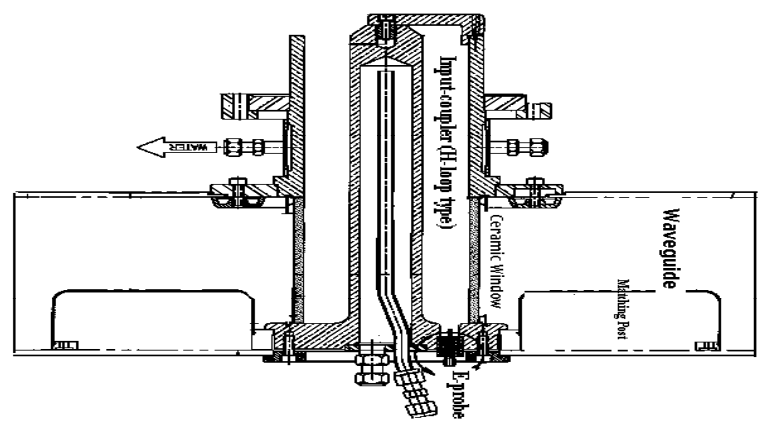

Figure 2: The ceramic window, where the rf power transmits through input coupler, showing the e-probe at the bottom near the window.

The electronic block diagram of the e-detector is shown in Figure 3. The rf lowpass filter is used to prevent interference with the $352-\mathrm{MHz}$ and any other HOM frequencies. The incoming signal is obtained while applying the $30-\mathrm{V}$ bias voltage in the circuit. An instrumentation amplifier amplifies the incoming signal, and then the current is converted to a voltage. The voltage signal passes through a buffer integrated circuit and finally reads into another amplifier circuit that sends the output to "signal out" or compares with the trip setting.

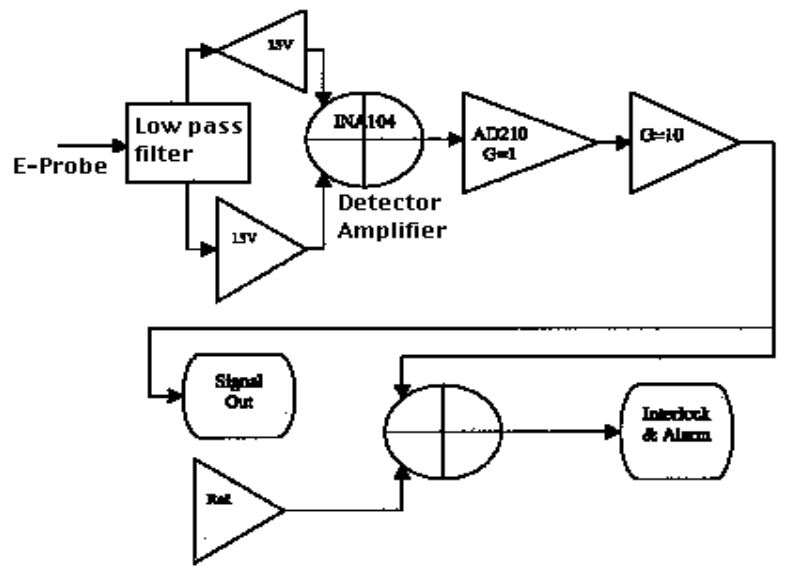

Figure 3: The block diagram of the e-detector board.
The signal output is read on a Sun workstation after it has been routed through an Alan Bradley circuit with EPICS codes. An output signal will be sent to the safety interlocks, once a reasonable trip point is determined.

\section{INITIAL MEASUREMENT}

\subsection{X-ray-induced Multipacting}

The most troublesome area due to multipacting was located in sector 36 cavity \#2 (S36 C2). In the past three years of operation, the input coupler/ceramic window was replaced three times. E-detectors designed at APS were installed on the sector 36 cavities (one per cavity). A SEE current was measured during rf conditioning as well as during injection and stored beam. Although there was no signal from any of the e-detectors during the rfconditioning, the e-detector recorded 6 volts from cavity \#2 with stored beam at $100 \mathrm{~mA}$, as shown in Figure 4.

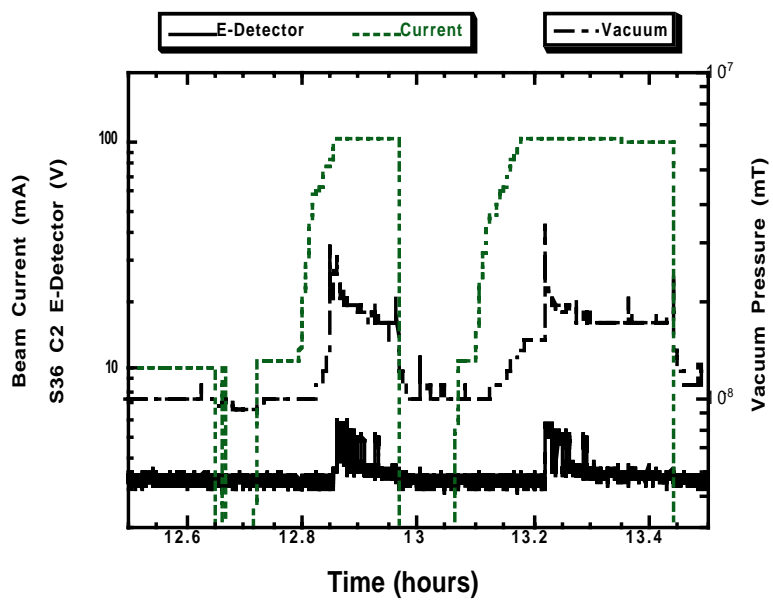

Figure 4: E-detector signal at sector 36 cavity \#2; the stored beam current and vacuum pressure are also shown.

As shown, the vacuum pressure follows the e-detector signal. However, the SEE current appears to be too high. Later, it was found this was due to the synchrotron radiation from the upstream bending magnet (S35BM-B) hitting the waveguide, where the ceramic window was located in this particular cavity (see Figure 5).

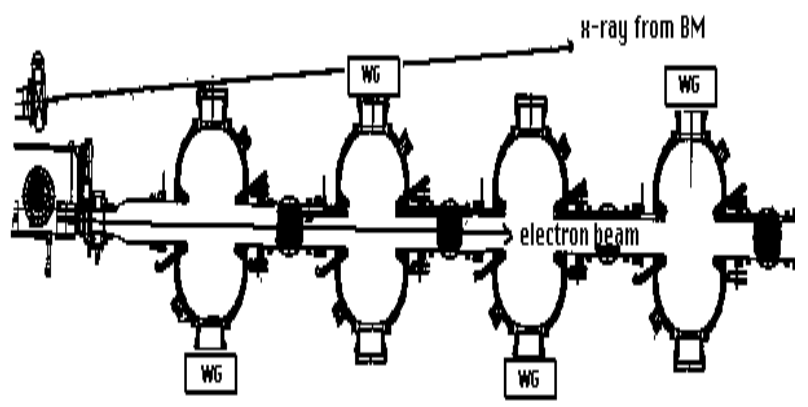

Figure 5: The layout of the sector 36 cavities, where the cavity \#2 waveguide is in the line of sight of the $\mathrm{x}$-ray trajectory from the upstream bending magnet. 
It appeared that the end absorber (copper block) at the end of the antechamber was not thick enough to block the $\mathrm{x}$-rays completely. After the transmitted x-ray dose was measured, more lead blocks were added ( 8 inches or 200 $\mathrm{mm}$ total) to prevent the $\mathrm{x}$-ray from penetrating the ceramic. The corresponding $\mathrm{x}$-ray dose calculation, shown in Figure 6 , shows that 8 inches of lead shielding was required to block the $\mathrm{x}$-rays.

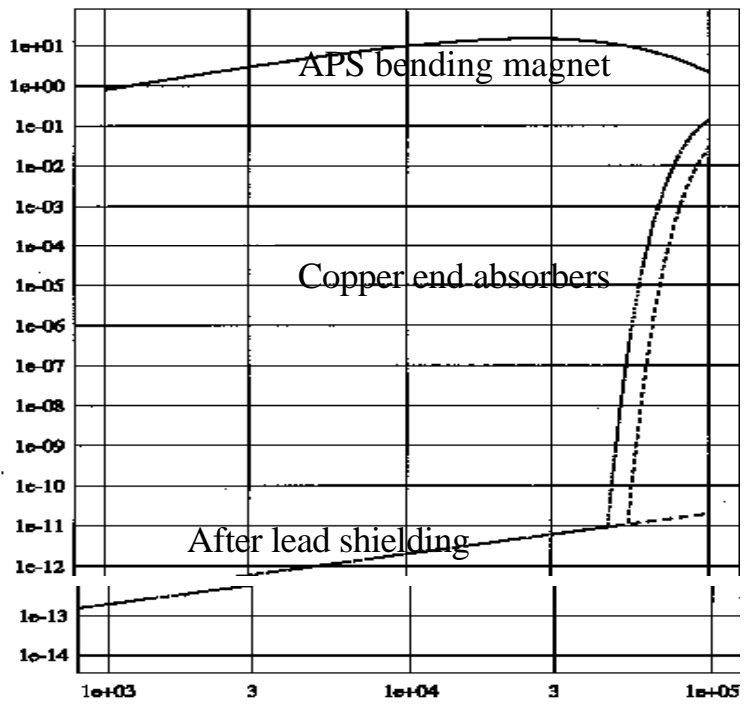

Energv (eV) vs Intensitv (watts/horizontal $\mathrm{cm}$ ) Figure 6: Calculation of transmitted x-ray energy from the APS bending magnet, shown with and without the lead shielding.

\subsection{Beam-induced Multipacting}

After the lead shielding was installed and the input coupler was replaced, the measurement continued. A typical result with a 100-mA beam is shown in Figure 7. The top line represents the stored beam current. The e-detector signal from the S36 C2 cavity appears to be a step-like pulse. The pulsed signal from the \#2 cavity e-detector repeats at about 80 seconds and stays at maximum value for 20 seconds. The signal due to the multipacting current is a series of short pulses on the order of nanoseconds. The pulse period was confirmed later in separate measurements with a fast sampling scope. This time scale is comparable with a gap of a few $\mathrm{cm}$ and an operating rf frequency of $352 \mathrm{MHz}$. The bottom of the step-like pulse from S36 C2 is overlapped with the S36 C1 cavity edetector signal, which is basically zero volts (noise signal in the middle of the graph).

The pulse width appears to be longer and higher as beam current and/or rf power increases in the cavity. In fact, when the beam current later reached more than 95 $\mathrm{mA}$, the signal saturated. Also shown is the vacuum pressure in the cavity (shown at the bottom of the graph). The pressure change is very small, which indicates local outgassing in the cavity. This is consistent with the SEE current from the e-detector. The ceramic temperature, taken from the upstream and downstream ceramic windows with infrared cameras, increases after the electron is bombarded on the surface (zigzag curves as shown).

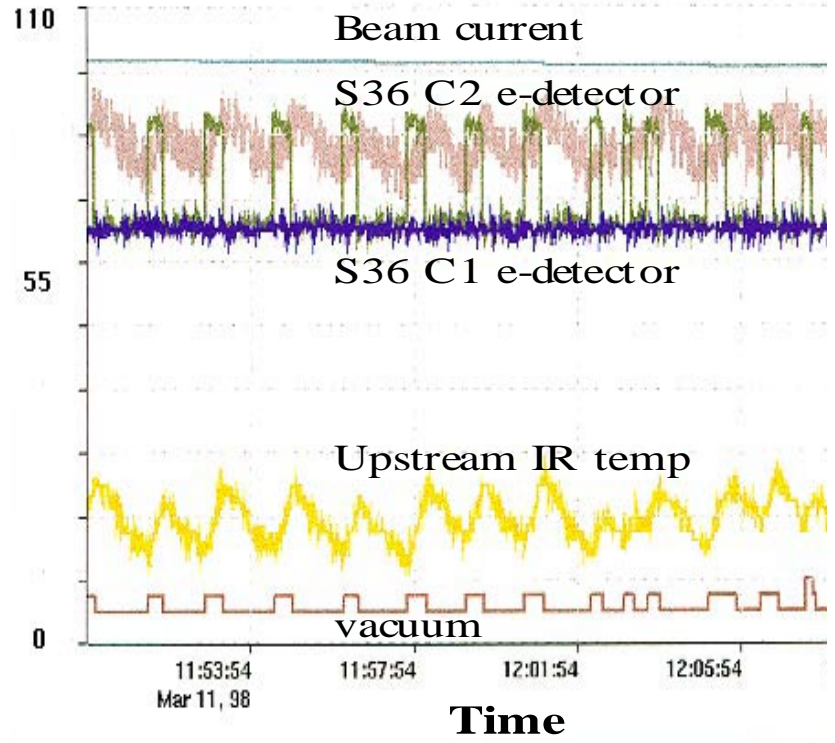

Figure 7: Electron detector signal from the newly replaced cavity. Also shown are vacuum pressure and ceramic temperature.

\subsection{Progress and Further Works}

- The prototype e-detector was tested, installed, and shown to respond to the multipacting current under various conditions.

- Recently, more emphasis is given to determine the SEE current using a multipacting simulation code developed by the University of Helsinki [4].

- More systematic measurements will be made during if conditioning once construction of the rf test-bed (the independent 350-MHz rf conditioning system) has been completed.

\section{ACKNOWLEDGMENTS}

The author would like to thank D. Proch at DESY for use of their e-detector design in early development stage. Many thanks also go to the students, especially W. Dower and $\mathrm{W}$. $\mathrm{Wu}$, for their technical assistance.

\section{REFERENCES}

[1] R.A. Kishek et al., "Steady state multipactor and dependence on material properties," Phys. Plasmas, V4 (3), 863 (1988).

[2] D. Proch et al., "Measurement of multipacting currents of metal surfaces in rf fields," Proc. of the 1995 Particle Accelerator Conference, 1776 (1996).

[3] K. Primdahl et al., "Reduction of multipactor in rf ceramic windows using a simple titanium-vapor deposition system," Proc. of the 1995 Particle Accelerator Conference, 1687 (1996).

[4] E. Somersalo et al., "Computational methods for analyzing electron multipacting in rf structures," Particle Accelerators, V59, 107 (1998). 